\title{
Berichterstattung über die Arbeitsgruppe „Klassifikator“ für die Periode Mai 1999 bis Mai 2000
}

\author{
S. Grafe
}

\section{Zusammenfassung}

Es darf zusammenfassend festgestellt werden, dass die Auswertung des Spuren- und Mengenelementegehalts im Blut nach Operationen am Knochen durch mathematische Muster eine gute Möglichkeit darstellt, Patientengruppen zu erfassen, die vergleichbar mit der Aussage des Wetterdienstes über die Lage von Hochs und Tiefs auf einer Wetterkarte eine prognostische Einschätzung nötiger Maßnah- men ermöglicht. Die Spurenelemente leisten so einen wichtigen Beitrag. Zentrale Aufgabe ist jetzt die Kostensenkung der Untersuchungsmethode durch Entwicklung der Hardware des Massespenktrographen mit einer Software zur Musterbestimmung, so dass jedes Großlabor unter Einsatz angelernter Laborantinnen oder Laboranten die Auswertung vornehmen kann. Die Vorbereitungen dazu sollen im kommenden Jahr anlaufen.
Die Arbeit der Arbeitsgruppe „Klassifikator" begründet sich auf einen Studienauftrag, das Verhalten von Mineralstoffen und Spurenelementen im menschlichen Körper bei der Ausnahmesituation des Vorliegens eines Knochenbruches und der danach erforderlichen Operation unter Einbringung von Metallimplantaten zu untersuchen. Ziel war die Antwort auf die Fragestellung: „Kann man den Serumgehalt der Spuren- und Mengenelemente nutzen, um frühzeitig die Patienten herauszufinden, die postoperativ in einer abweichenden akuten Phasereaktion zu erkennen geben, ob sie eine entzündliche Komplikation entwickeln, die Ausgangspunkt für eine Infektion werden kann."

Von Anbeginn an war es den Beteiligten klar, dass auch bei heute möglicher exakter Bestimmung der Serumspiegel einzelner Elemente dazu keine einfache Aussage gemacht werden kann. Die Bestimmungen wurden mit einem Massenspektrographen vorgenommen, der nach den Richtlinien der Bundesärztekammer (RiliBÄk) auf seine Qualitätsmerkmale hin

OP-JOURNAL 2000; 16: 302-304

(c) Georg Thieme Verlag Stuttgart · New York laufend überprüft wird. Von Beginn an waren folgende Probleme permanent.

a Abhängigkeit der Spuren- und Mengenelementegehalte im Blutplasma von Ernährung und Ausscheidung.

- Wirksamkeit von Speichern dieser Elemente im Körper.

- Interaktionen von Elementen bei bereits bekannten Einzelwirkungen bzw. -aufgaben.

- Veränderungen der Serumspiegel von Elementen durch Reaktion auf auftretende Bakterien oder Noxen.

Für die Beteiligten galt es von Anbeginn an einzukalkulieren, dass zur Lösung der Aufgabe nur Deutungen in Frage kommen. Der Möglichkeit der Verwendung der Unschärfe von Parametern wurden die größten Chancen eingeräumt. Aus der Mathematik sind Mustererkennungsmethoden bekannt, die durch computertechnische Eingrenzungen die Messparameter unscharf modellieren können. Ihr Vorteil gegenüber einer Grenzwertbestimmung beruht auf der Möglichkeit auch multikausal beeinflusste Messgrößen durch Betrachtung der Eigenschaften mehrerer Faktoren zu einer Aussage heranzuziehen. Nachdem im Zeitraum von März 1995 bis März 1998 ein auf Mustererkennung der Spurenelementeveränderungen begründeter
Klassifikationsprozess angelernt und geprüft worden war, wurde der Klassifikator in die klinische Praxis des Ev.Luth. Diakonissenkrankenhauses Leipzig gGmbH eingeführt. Die Kostspieligkeit der Untersuchung ließ das Scrining nur bei einer ausgewählten Gruppe von Patienten zu. Als geeignet und als besonders markant empfahl sich die Gruppe der proximalen Oberschenkelbrüche. Im Zeitraum von März 1998 bis März 1999 stand eine wichtige Problemstellung an. Es sollten Vergleiche von bakteriellen Abstrichbefunden mit den Klassifikationsergebnissen gezogen werden. Dabei konnten wichtige Hinweise zur Deutung der Mustererkennungsergebnisse für die prognostischen Aussage gegeben werden. Die Abbildung 1 zeigt deutlich, dass es einerseits einen Zusammenhang der Häufigkeit von Wundkontaminationen bei der Operation mit der Häufigkeit der positiven Klassifikation gegeben hat und dass andererseits die Häufigkeit der Nichterfassung entzündlicher Reaktionen bei sekundärem Keimnachweis beobachtet werden kann. Gleichzeitig konnte festgestellt werden, dass mit $\mathrm{Zu}-$ nahme der Liegezeit von Drainagen die sekundäre Kontamination zunahm.

Die gleichzeitig durch die Ergebnisse einer Promotionsarbeit (Khader, Th.) ausgewiesene Tatsache, dass bei einer Reihe von Elementen eine höhere Signifikanz der Veränderung am 2.postoperativen Tag zu beobachten war, hat uns veranlasst, in der darauf folgenden Untersuchungsserie ab April 1999 wie folgt zu verfahren:

1. Entfernung der Wunddrainage nach der Operation am Ende des 1. postoperativen Tages und

2. Abnahme des Serums für die Elementemuster am zweiten postoperativen Tage.

Ein Nachteil dieser Änderung des Verfahrens wurde nicht beobachtet. Untersucht wurden 160 Patienten, davon waren 140 proximale Oberschenkel- und 20 Unter- 


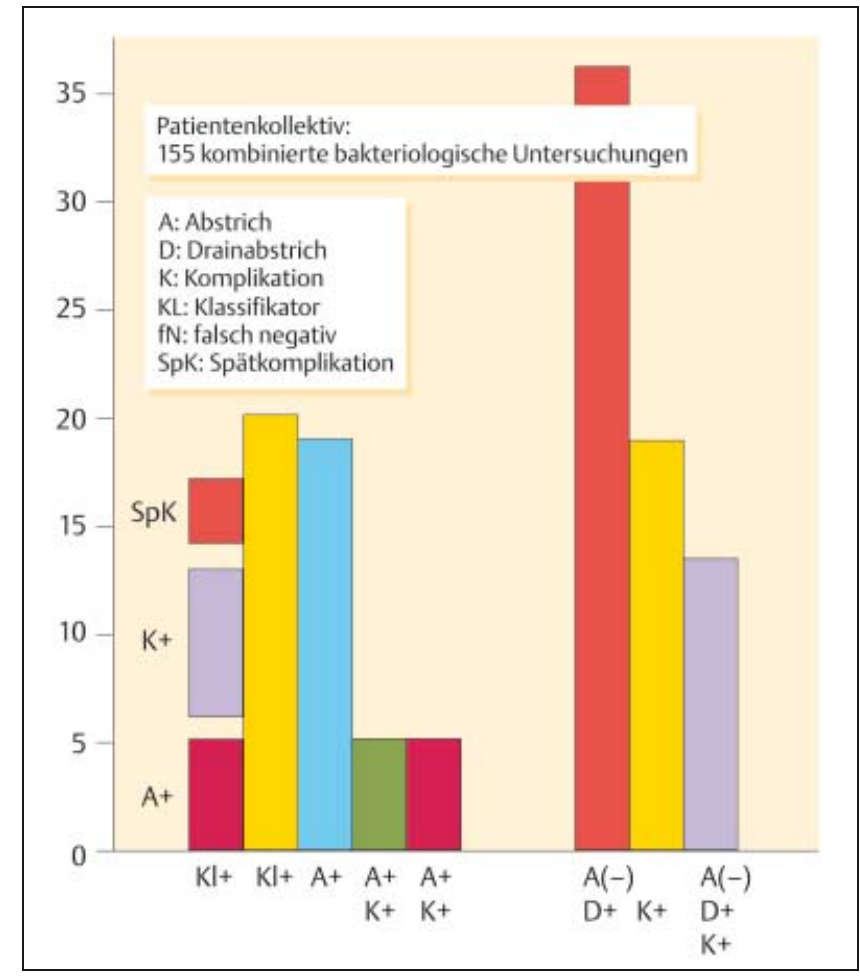

Abb.1 Patientenkollektiv von 155 kombinierten bakteriologischen Untersuchungen. Gegenüberstellung: Infektionen und Klassifikationen.

schenkelfrakturen. 72 bzw. 68 davon entwickelten Auffälligkeiten, wobei das zurückgelassene Hämatom kaum eine Rolle spielte. An vorderster Stelle wurden sonographisch sicht- bzw. messbare secundäre Flüssigkeitsansammlungen (Abb.2) meist sogenannte Serome nachgewiesen. Die fast deckungsgleichen Verläufe von positiv klassifizierten Patienten mit den nachgewiesenen postoperativen Auffälligkeiten zeigt Abb.3. 14 Patienten entwickelten Auffälligkeiten, die nach den strengen Kriterien der Arbeitsgruppe Infektiologie der DAO als Infektion eingestuft wurden, 13 davon am proximalen Oberschenkel (8,8 bzw. 9,3\%). Aufgrund der Tatsache, dass Patienten, die ein positives Muster aufwiesen $(n=27)$, bereits nach einem noch zu nennenden Algorithmus bei uns behandelt wurden, kann man aus der gleichen Verteilung der Infektionen: sieben $\mathrm{zu}$ sieben keine Schlüsse ziehen. Auffällig bleibt die zunächst noch nicht mathematisch abzuklärende Tatsache, dass einerseits die Sommermonate Mai bis August 1999 kaum falschnegative Befunde aufwiesen, diese sich andererseits aber vorwiegend auf die Monate September bis März verteilten (Abb.4). Diese jahreszeitliche Trift muss mathematisch noch erfasst werden. Ob sie mit der allgemeinen Jahrestrift der Konzentrationswerte zu erklären ist,

Abb. 3 Monatliche Gegenüberstellung der Infektionen, auffälligen Frühbefunden und negativen Klassifikationsergebnissen.

Abb. 4 Monatliche Gegenüberstellung der Infektionen, auffälligen Frühbefunden und positiven Klassifikationsergebnissen.

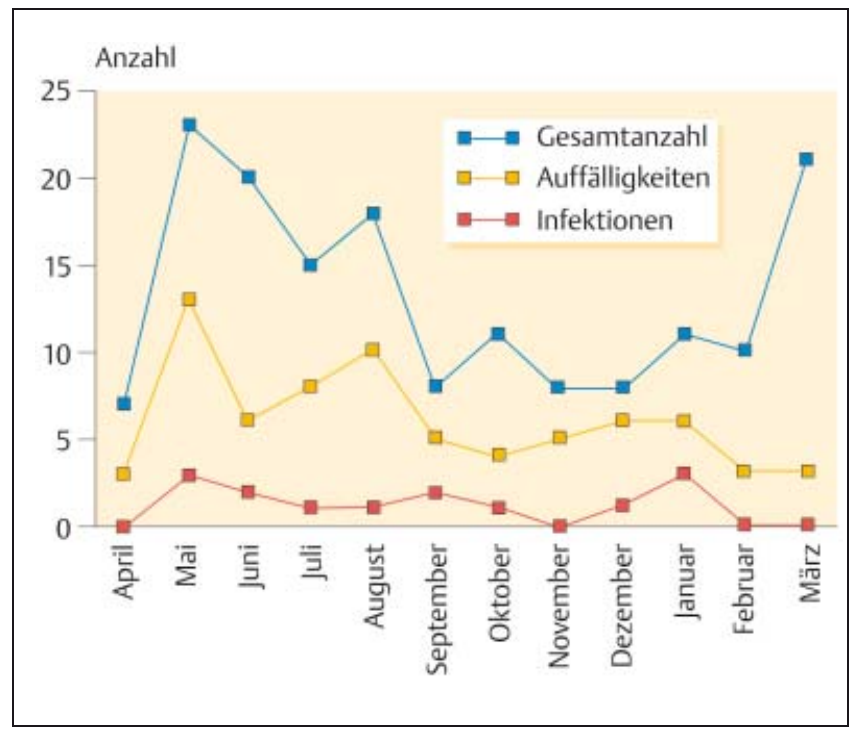

Abb. 2 Monatliche Gegenüberstellung der Infektionen zu den klinisch auffälligen Frühbefunden.
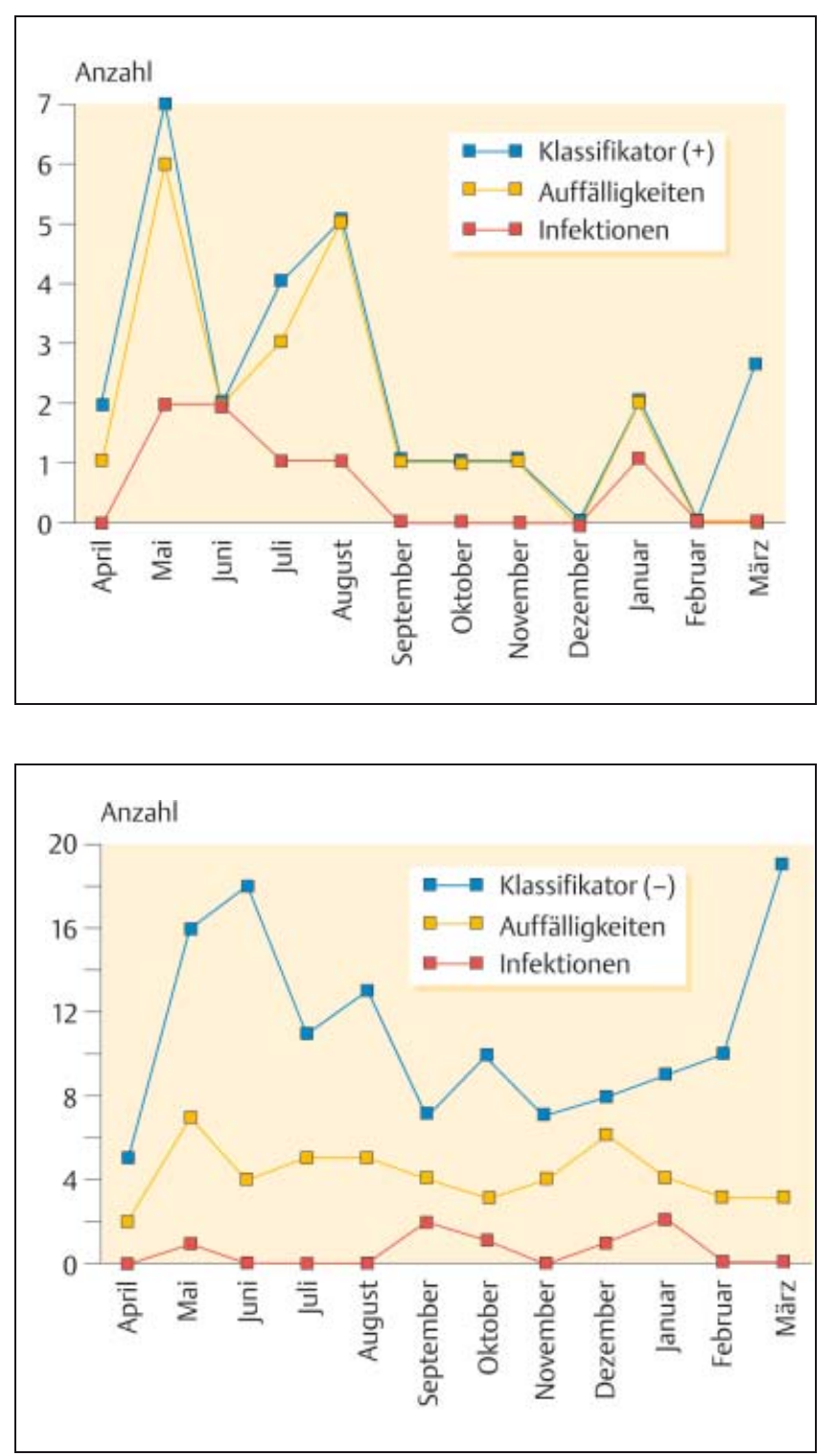


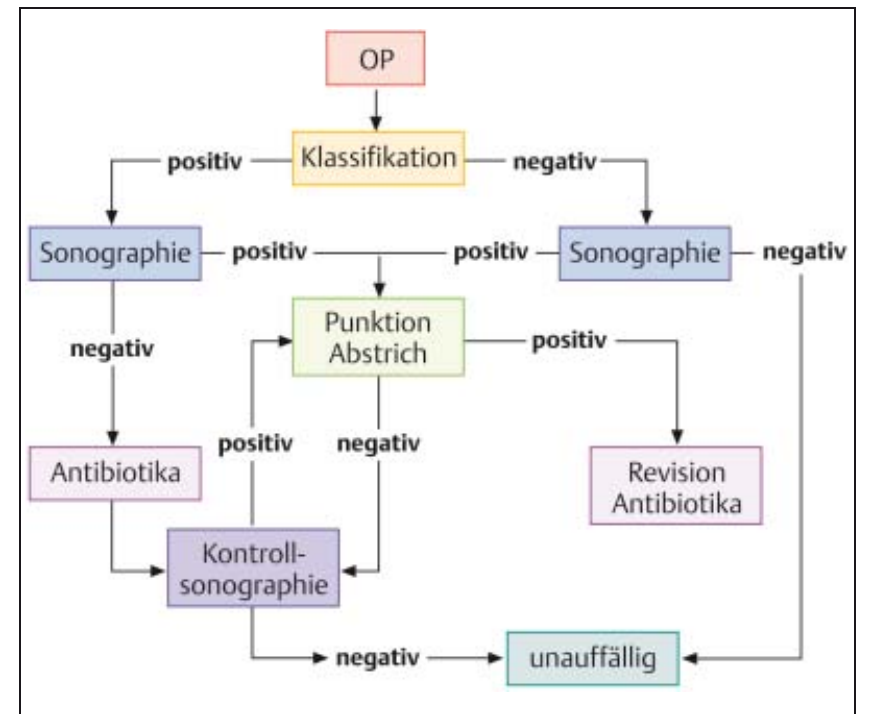

Abb.5 Algorithmus für die Nutzung der Klassifikation.

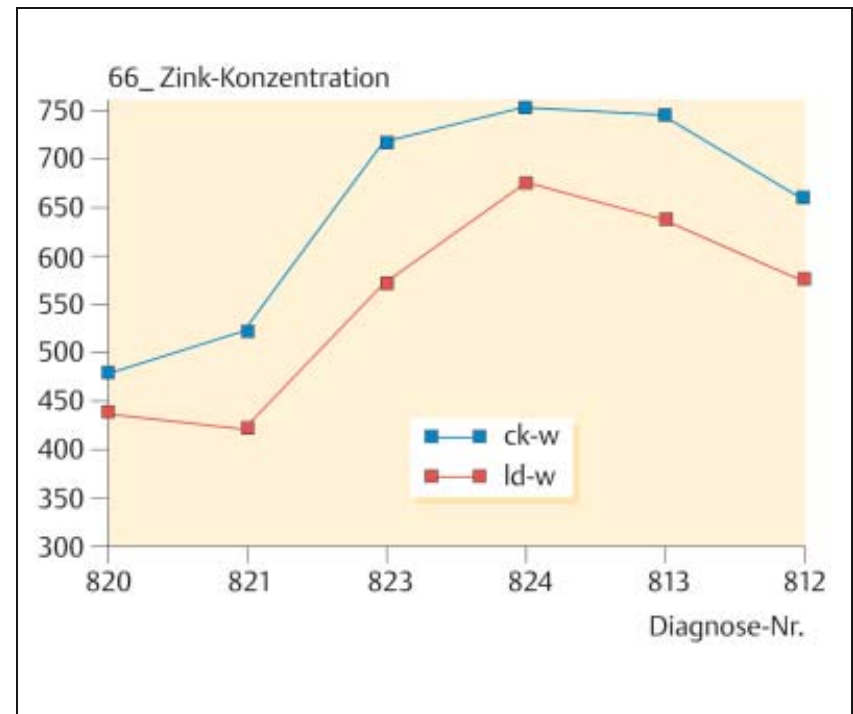

Abb. 6 Korrekturfaktor der Zinkkonzentrationen im Serum der Patienten des Küchwaldklinikums Chemnitz. muss zunächst also noch offen bleiben. Deutliche Korrelation zeigen die Zahlen der positiven Muster mit der Entwicklung von Auffälligkeiten, die auch im Durchschnitt die größeren Flüssigkeitsabsonderungen betrafen $\left(11 / 14 \mathrm{~cm}^{3}\right)$. Eine der Erwartung entsprechende $\mathrm{Ab}$ nahme der falschnegativen Befunde trat zwar ein. Bei der kleinen Zahl ist eine Signifikanzaussage aber nicht möglich gewesen (7/5). Ungeachtet dessen ist die Tatsache von Bedeutung, dass die Güte des negativen Vorhersagewertes, die Anwendung des Klassifikators zur Steuerung des therapeutischen Verhaltens im Leipziger Diakonissenhaus nach folgendem Algorithmus erlaubt (Abb.5).

Weitere Schwerpunkte in der Studie zur Mustererkennung von Spuren- und Mengenelementen lagen in der Hand anderer Kliniken.

Erfolgreich ist die Arbeit in der Unfallchirurgischen Universitätsklinik des Bergmannsheils Bochum fortgesetzt worden. Sie beruhte auf der Möglichkeit, dass die mathematische Adaption der Muster in Bochum an die Urform im Diakonissenhaus Leipzig sichergestellt werden konnte. Die Schlussfolgerungen aus den dort vorliegenden Ergebnissen sind auch aus ökonomischer Sicht erfolgver- sprechend. Der Veröffentlichung der Ergebnisse kann man mit Interesse entgegen sehen. Studien aus dem Unfallchirurgischen Klinikum Küchwald in Chemnitz konnten bereits im vergangenen Jahr den für diesen Ort errechneten Korrekturfaktor bestätigen (Abb.6).

Als Schlussfolgerung darf zusammenfassend festgestellt werden, dass die Auswertung der Spuren- und Mengenelementegehalts im Blut nach Operationen am Knochen durch mathematische Muster eine gute Möglichkeit darstellt, Patientengruppen $\mathrm{zu}$ erfassen, die vergleichbar mit der Aussage des Wetterdienstes über die Lage von Hochs und Tiefs auf einer Wetterkarte eine prognostische Einschätzung nötiger Maßnahmen ermöglicht. Die Spurenelemente leisten so einen wichtigen Beitrag. Zentrale Aufgabe ist jetzt die Kostensenkung der Untersuchungsmethode durch Entwicklung der Hardware des Massespektrographen mit einer Software zur Musterbestimmung, so dass jedes Großlabor unter Einsatz angelernter Laborantinnen oder Laboranten die Auswertung vornehmen kann. Die Vorbereitungen dazu sollen im kommenden Jahr anlaufen

- Spuren- und Mengenelemente besitzen Schaltstellen im Stoffwechsel des Menschen.
- Aussage über Elemente, die im Rahmen der Infektabwehr und im Knochenstoffwechsel Aufgaben übernehmen, sind geeignet Fraktursituationen, Knochenoperationsstress und die Akute-Phase-Reaktion nach Keimkontakt zu beschreiben.

- Ihre Serumgehalte ermöglichen es, Beziehungen darzustellen, die eine Gruppenklassifizierung im Hinblick auf die Abwehrlage am 2. Tag nach Knochenbruchoperationen ermöglichen.

- Mathematische Modelle, die unter Einbeziehung der Unschärfe Mustererkennung zulassen, werden durch den Computer erstellt.

- Die Bestimmung der Elemente nach Knochenoperationen mit dem Massenspektrographen kann durch ihre Beziehungsmuster zur Klassifikation genutzt werden.

\section{Literatur}

${ }^{1}$ Khader Th. Verhalten der Spurenelemente als Antwort auf eine Osteosynthese-Operation, Dissertation Medizinische Fakultät Leipzig, April 2000

Prof. Dr. med. S. Grafe

Leitender Chefarzt

Ev. Diakonissenkrankenhaus

Leipzig gGmbH

Georg-Schwarz-Str. 49

04177 Leipzig 